\title{
Evaluación del comportamiento estructural y de resistencia a la corrosión de armaduras de acero inoxidable austenítico AISI 304 y dúplex AISI 2304 embebidas en morteros de cemento Pórtland ${ }^{(*)}$
}

\author{
E. Medina*, A. Cobo* y D.M. Bastidas**
}

Resumen

\begin{abstract}
Se ha evaluado el comportamiento mecánico y estructural de dos aceros inoxidables corrugados, el austenítico EN 1.4301 (AISI 304) y el dúplex EN 1.4362 (AISI 2304), y se han comparado con el tradicional acero al carbono B500SD. El estudio se ha realizado en tres niveles: a nivel de barra, de sección y de pieza. Las diferentes características mecánicas de los aceros inoxidables condicionan el comportamiento a nivel de sección y de pieza estructural. El estudio del comportamiento frente a la corrosión de los dos aceros inoxidables se ha realizado mediante mediciones electroquímicas monitorizando el potencial de corrosión y la resistencia de polarización de armaduras embebidas en probetas de mortero contaminado con diferentes concentraciones de cloruros durante un tiempo de exposición de un año. Ambos aceros inoxidables permanecen en estado pasivo en las probetas para todos los contenidos de cloruros.
\end{abstract}

\section{Evaluation of structural behaviour and corrosion resistant of austenitic AISI 304 and duplex AISI 2304 stainless steel reinforcements embedded in ordinary Portland cement mortars}

\begin{abstract}
The mechanical and structural behaviour of two stainless steels reinforcements, with grades austenitic EN 1.4301 (AISI 304) and duplex EN 1.4362 (AISI 2304) have been studied, and compared with the conventional carbon steel B500SD rebar. The study was conducted at three levels: at rebar level, at section level and at structural element level. The different mechanical properties of stainless steel directly influence the behaviour at section level and structural element level. The study of the corrosion behaviour of the two stainless steels has been performed by electrochemical measurements, monitoring the corrosion potential and the lineal polarization resistance (LPR), of reinforcements embedded in ordinary Portland cement (OPC) mortar specimens contaminated with different amount of chloride over one year time exposure. Both stainless steels specimens embedded in OPC mortar remain in the passive state for all the chloride concentration range studied after one year exposure.
\end{abstract}

Keywords

Stainless steel; Reinforcements; Corrosion; Chloride; Ductility.

\section{INTRODUCCIÓN}

La característica que singulariza el corrugado de acero inoxidable frente al de acero al carbono es su excelente resistencia a la corrosión, especialmente la desencadenada por los iones cloruro. Los aceros inoxidables austeníticos y dúplex son los dos tipos recomendados para su utilización en el hormigón. El austenítico es el más utilizado y es conocida su resistencia a la corro- sión en hormigones con gran contenido en cloruros. Su tolerancia a dichos cloruros es de 5 a 10 veces superior a la del acero al carbono ${ }^{[1]}$. El acero inoxidable dúplex es más económico que el austenítico por su menor contenido de níquel, y además, presenta una mayor resistencia a la corrosión por picaduras de cloruros $^{[2]}$. En todos los casos, los aceros inoxidables permiten rebajar unas medidas de durabilidad del hormigón pensadas, en su origen, para proteger el acero

(·) Trabajo recibido el día 30 de enero de 2012 y aceptado en su forma final el día 5 de junio de 2012.

Escuela Universitaria de Arquitectura Técnica, Universidad Politécnica de Madrid (UPM). Avda. Juan de Herrera 6, 28040 Madrid.

Centro Nacional de Investigaciones Metalúrgicas, CENIM-CSIC. Dpto. Ingeniería de Superficies, Corrosión y Durabilidad. Avda. Gregorio del Amo 8, 28040 Madrid, España. E-mail: david.bastidas@cenim.csic.es. 
al carbono, como son los recubrimientos mínimos y las aberturas de fisura máximas ${ }^{[3}$ y 4$]$.

Además, diferentes estudios demuestran que el uso combinado de barras de acero inoxidable y acero al carbono en la armadura de una misma estructura de hormigón no aumenta el riesgo de corrosión de dicha armadura en comparación con otra únicamente de acero al carbono, incluso cuando dichas barras están en contacto eléctrico directo ${ }^{[5}$ y 6].

Por lo tanto, la utilización de armaduras de acero inoxidable, del tipo austenítico o dúplex, en toda la estructura o sólo en algunos de sus elementos que vayan a estar expuestos a ambientes agresivos, especialmente con presencia de iones cloruro, mejora notablemente la durabilidad de la estructura, por lo que es una alternativa que está recibiendo cada vez más consideración a pesar de su alto coste inicial en comparación con el acero al carbono.

Sin embargo, la utilización de armaduras de acero inoxidable es escasa y no se tiene conocimiento con detalle de su comportamiento mecánico y estructural, mientras que sí que se tiene del acero al carbono. También son escasos los estudios frente a la corrosión de armaduras de acero inoxidable embebidas en probetas de mortero contaminado con cloruros ${ }^{[7]}$.

En este trabajo se ha analizado a nivel de barra corrugada, de sección de viga de hormigón y de pieza de hormigón armado, el comportamiento mecánico, de ductilidad y estructural de dos armaduras de acero inoxidable, una austenítica AISI 304 y otra dúplex AISI 2304, comparándolas con la tradicional de acero al carbono B500SD. También se ha evaluado la resistencia a la corrosión de las armaduras inoxidables AISI 304 y AISI 2304 embebidas en probetas de mortero contaminado con diferentes concentraciones de cloruros, mediante técnicas electroquímicas, en ensayos durante un año.

\section{MÉTODO EXPERIMENTAL}

Para evaluar el comportamiento mecánico y estructural de los aceros inoxidables corrugados, se han com- parado dos tipos, el austenítico AISI 304 (EN 1.4301) y el dúplex AISI 2304 (EN 1.4362), cuya composición química se recoge en la tabla I, junto a la del acero al carbono B500SD. El estudio se ha realizado a nivel de barra, de sección y de pieza.

A nivel de barra, el estudio se ha desarrollado experimentalmente, ensayando a tracción barras corrugadas de diferentes diámetros de acuerdo a las normas UNE-EN 10002-1 y UNE-EN ISO 15630-1[8 y 9], y analíticamente, estudiando los parámetros de ductilidad de cada uno de los aceros ensayados. Para los ensayos se eligieron los diámetros nominales de 8 , 16 y $20 \mathrm{~mm}$, el primero laminado en frío y los otros dos laminados en caliente. Se han ensayado dos probetas de cada tipo.

A partir de los resultados obtenidos en los ensayos de tracción, se han calculado los parámetros de ductilidad de los tres aceros, de acuerdo a los siguientes criterios:

(a) El establecido en diferentes normativas europeas, concretamente la instrucción EHE-08, el Código Modelo 90 (CM90), y los Eurocódigos 2 y 8 (EC2 y EC8) ${ }^{[10-13]}$, mediante los dos parámetros siguientes: la relación entre la carga de rotura y el límite elástico o ratio de endurecimiento a tracción $\left(\mathrm{f}_{\mathrm{s}} / \mathrm{f}_{\mathrm{y}}\right)$ y la deformación para la carga máxima $\left(\varepsilon_{\text {máx }}\right)$.

(b) El concepto de acero equivalente según el parámetro p de Cosenza (Ec. (1)), el área $A_{\text {nom }}$ definido por Creazza (Ec. (2)) y el índice de tenacidad $I_{d}$ de Ortega (Ec. (3)) ${ }^{[14]}$.

$$
\begin{gathered}
p=\left(\frac{f_{s}}{f_{y}}-1\right)^{0,9}\left(\varepsilon_{\text {máx }}+3 \varepsilon_{s h}\right)^{0,75} \\
A_{\text {nom }}=\frac{2}{3}\left(\varepsilon_{\text {máx }}-\varepsilon_{y}\right)\left(f_{s}-f_{y}\right)
\end{gathered}
$$

$$
\mathrm{I}_{\mathrm{d}}=\left(1+\frac{\mathrm{f}_{\mathrm{s}}}{\mathrm{f}_{\mathrm{y}}}\right)\left(\frac{\varepsilon_{\text {máx }}}{\varepsilon_{\mathrm{y}}}-1\right)
$$

Tabla I. Composición química de los aceros ensayados (\% peso, resto Fe)

Table I. Chemical composition of the tested steels (\% weight, balance Fe)

\begin{tabular}{lcccccccccc}
\hline Acero & $\% \mathbf{C}$ & $\% \mathbf{S i}$ & $\% \mathbf{M n}$ & $\% \mathbf{P}$ & $\% \mathbf{S}$ & $\% \mathbf{C r}$ & $\% \mathbf{N i}$ & $\% \mathbf{C u}$ & $\% \mathbf{~ N}$ & $\% \mathbf{M o}$ \\
\hline AISI 304 & 0,07 & 0,75 & 2,00 & 0,075 & 0,015 & 18,50 & 9,00 & - & - & - \\
AISI 2304 & 0,03 & 1,00 & 2,00 & 0,035 & 0,010 & 23,00 & 3,50 & 0,20 & 0,10 & 0,20 \\
B500SD & 0,45 & 0,22 & 0,72 & $<0,01$ & 0,022 & 0,13 & 0,13 & 0,18 & - & - \\
\hline
\end{tabular}


donde $\varepsilon_{\mathrm{sh}}$ es la deformación unitaria al final del escalón de cedencia (en su caso) y $\varepsilon_{\mathrm{y}}$ la deformación en el límite elástico.

(c) La propuesta del CEB (Comité EuroInternational du Béton) de una nueva clasificación de ductilidad del acero, basándose en la formulación de Cosenza, que para el de alta ductilidad o Clase S establece los siguientes límites ${ }^{[15]}$ :

$$
\begin{aligned}
& \left(\frac{f_{s}}{f_{y}}-1\right)_{k} \geq 0,13 \quad \text { y } \quad \varepsilon_{\text {máx }} \geq 9 \% \\
& \left(\frac{f_{s}}{f_{y}}-1\right)_{k} \geq 0,15 \quad \text { y } \quad \varepsilon_{\text {máx }} \geq 6 \% \\
& \left(\frac{f_{s}}{f_{y}}-1\right)_{k} \geq 0,17 \quad \text { y } \quad \varepsilon_{\text {máx }} \geq 5 \%
\end{aligned}
$$

Respecto al estudio a nivel de sección, se han elaborado por iteración los diagramas momento-curvatura $(M-\varphi)$ de dos secciones de vigas tipo, una viga plana de $50 \times 30 \mathrm{~cm}$ y otra de canto de $30 \times 50 \mathrm{~cm}$, ambas armadas con diferentes cuantías de armadura de acero al carbono y de acero inoxidable dúplex (Tabla II).

Se ha seleccionado el acero inoxidable dúplex por tener las mismas características mecánicas que el acero inoxidable austenítico y sin embargo ser más económico por su menor contenido en níquel. A par- tir de los diagramas obtenidos, se han comparado entre sí los valores de ductilidad de la sección armada con ambos aceros.

Para el estudio a nivel de pieza, se han fabricado cuatro vigas de hormigón de 10 x 15 cm de sección y $1 \mathrm{~m}$ de longitud entre apoyos, armadas con cercos de barra corrugada de diámetro $\varnothing 8 \mathrm{~mm}$ colocados a $45^{\circ}$ y a $7 \mathrm{~cm}$ en las vigas 1 y 3 , y a $10 \mathrm{~cm}$ en las vigas 2 y 4 armadas longitudinalmente de la siguiente forma:

(a) Viga 1: dos barras corrugadas de diámetro $\varnothing 8$ de acero al carbono.

(b) Viga 2: dos barras corrugadas de diámetro $\varnothing 16 \mathrm{~mm}$ de acero al carbono.

(c) Viga 3: dos barras corrugadas de diámetro $\varnothing 8$ de acero dúplex AISI 2304.

(d) Viga 4: dos barras corrugadas de diámetro $\varnothing 16$ mm de acero dúplex AISI 2304.

Las vigas se han ensayado hasta la rotura a flexión, aplicando la carga en dos puntos equidistantes a un tercio de la longitud de la viga, y se ha comprobado el comportamiento a desplazamiento y resistencia por medio de los diagramas carga-desplazamiento.

Por último, para el estudio de la resistencia a la corrosión por cloruros, se han elaborado ocho probetas de mortero de $8 \times 5,5 \times 2 \mathrm{~cm}$ con cemento Pórtland 32,5 CEM II BL y arena normalizada CEN DIN-EN 196-1, con una dosificación de cemento, arena y agua de 1:3:0,5 respectivamente. En cada probeta se han embebido dos barras corrugadas de $8 \mathrm{~mm}$ de diámetro de acero inoxidable austenítico AISI 304 o de acero inoxidable dúplex AISI 2304. Las probetas se fabricaron sin adición de cloruros y añadiendo

Tabla II. Secciones de vigas consideradas en el estudio a nivel de sección

Table II. Sections beams included in the study at the section level

\begin{tabular}{cccc}
\hline $\begin{array}{c}\text { Vigas planas } \\
\mathbf{5 0} \mathbf{3 0} \mathbf{~ c m}\end{array}$ & $\begin{array}{c}\text { Vigas de canto } \\
\mathbf{3 0} \mathbf{5 0} \mathbf{~ c m}\end{array}$ & $\begin{array}{c}\text { Armadura a } \\
\text { tracción }\end{array}$ & $\begin{array}{c}\text { Cuantía de armadura respecto } \\
\text { a la sección de hormigón \%o }\end{array}$ \\
\hline P1 & C1 & $4 \varnothing 12^{*}$ & 3,02 \\
P2 & C2 & $3 \varnothing 16$ & 4,02 \\
P3 & C3 & $4 \varnothing 16$ & 5,36 \\
P4 & C4 & $5 \varnothing 16$ & 6,70 \\
P5 & C5 & $6 \varnothing 16$ & 8,04 \\
P6 & C6 & $7 \varnothing 16$ & 9,38 \\
P7 & C7 & $5 \varnothing 20$ & 10,47 \\
P8 & C8 & $6 \varnothing 20$ & 12,57 \\
P9 & C9 & $8 \varnothing 20$ & 16,76 \\
\hline
\end{tabular}

\footnotetext{
* Se corresponde con la cuantía mínima geométrica de armado que establece la instrucción EHE-08 para una viga de hormigón.
} 
$\mathrm{CaCl}_{2}$ en proporciones de $0,4,2$ y $4 \%$ en relación al peso de cemento. Las probetas se han curado durante 28 días al $95 \%$ de humedad relativa y $20^{\circ} \mathrm{C}$ en una cámara de curado modelo CM-200 de la marca Ibertex, y después se han mantenido en un recipiente hermético, con un $95 \%$ de humedad relativa, durante todo el periodo de tiempo en el que se han realizado los ensayos electroquímicos.

En la caracterización electroquímica, se ha utilizado un potenciostato-galvanostato EG\&G PARC, con una configuración de celda de tres electrodos: la barra corrugada como electrodo de trabajo, un electrodo de referencia de calomelanos (SCE), y un anillo de guarda de acero inoxidable de $7 \mathrm{~cm}$ de diámetro como contraelectrodo. En cada ensayo, estabilizado el potencial de corrosión $\mathrm{E}_{\text {corr }}$ transcurridos $20 \mathrm{~min}$, se ha aplicado el método de la resistencia de polarización lineal (LPR) para determinar la resistencia de polarizacion $\mathrm{R}_{\mathrm{p}}$, aplicando a cada barra una pequeña señal de potencial de $\pm 15 \mathrm{mV}$ respecto del $\mathrm{E}_{\text {corr }}$ a una velocidad de $0,1667 \mathrm{mV} / \mathrm{s}^{[16]}$. A partir de la $\mathrm{R}_{\mathrm{p}}$ se ha obtenido la intensidad de corrosión $\mathrm{i}_{\text {corr, }}$, según la ecuación (7), tomando el valor de $52 \mathrm{mV}$ para el coeficiente B de Stern-Geary ${ }^{[17]}$.

$$
i_{\text {corr }}=\frac{B}{R_{P}}
$$

También se ha calculado la $i_{\text {corr }}$ por la técnica de pulsos potenciostáticos, imponiendo a cada barra un pulso de potencial de $+50 \mathrm{mV}$ respecto del $\mathrm{E}_{\text {corr }}$ durante 2 s y registrando la caída de potencial que experimenta durante los $120 \mathrm{~s}$ siguientes. La carga adquirida por la doble capa electroquímica $\mathrm{C}$ se consume gradualmente en el proceso de corrosión, y el potencial tiende a volver al $\mathrm{E}_{\text {corr }}$ en un tiempo $t$, variando según la ecuación (8):

$$
\frac{\eta_{t}}{\eta_{0}}=e^{\left(\frac{-t}{C \cdot R_{p}}\right)}
$$

donde $\eta_{\circ}$ es la máxima polarización en el momento de interrumpir la corriente y $\eta_{t}$ la polarización en el tiempo $t$ desde que se interrumpió la corriente.

A partir del gráfico potencial-tiempo obtenido (Fig. 1), se ha calculado gráficamente la constante tiempo del proceso de corrosión, que es el producto $C \cdot R_{p}$, por la distancia desde el origen a la intersección del valor $0,37 \cdot \eta_{0}$ con la curva descendente, siendo

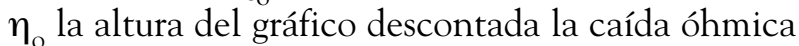
del hormigón ${ }^{[18]}$.

El valor de la capacidad de la doble capa electroquímica $\mathrm{C}$ que se ha asumido en los cálculos para obtener el valor de $R_{p}$ ha sido de $5 \mu \mathrm{F} / \mathrm{cm}^{2}$. A partir del valor de $R_{p}$ se determina el valor de $i_{\text {corr }}$ según la ecuación (7).

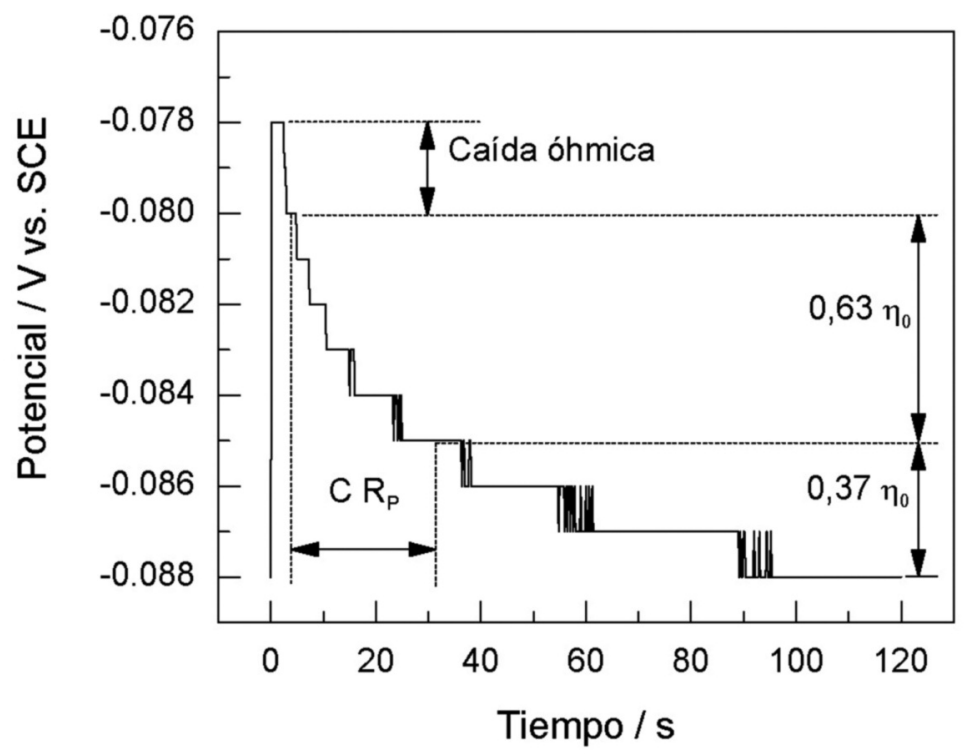

Figura 1. Gráfico Potencial-Tiempo obtenido al aplicar la técnica de pulsos potenciostáticos.

Figure 1. Potential-Time graph obtained by applying potentiostatic pulse technique. 


\section{RESULTADOS Y DISCUSIÓN}

\subsection{Ensayos a tracción de las armaduras}

Los resultados de los ensayos de tracción de las barras corrugadas de los tres aceros, se recogen en la tabla III (valores medios de las dos probetas ensayadas por cada diámetro y acero).

Se comprueba que la resistencia máxima $\mathrm{f}_{\mathrm{s}}$ y el límite elástico $\mathrm{f}_{\mathrm{y}}$ sólo presentan diferencias con motivo del proceso de fabricación de las barras, laminadas en caliente o en frío, y no por el tipo de acero con el que están elaboradas. Las barras laminadas en caliente de los tres aceros toman valores de resistencia en el límite elástico entre 507 y $609 \mathrm{~N} / \mathrm{mm}^{2}$, por lo que a efectos de cálculo estructural, el valor característico de $500 \mathrm{~N} / \mathrm{mm}^{2}$ que establece la instrucción EHE-08 para el acero al carbono B500SD parece también el adecuado para los aceros inoxidables. Respecto a las barras laminadas en frío $(\varnothing 8 \mathrm{~mm})$, las de acero inoxidable alcanzan valores un $50 \%$ más altos que las de acero al carbono.

En cambio, respecto al módulo de elasticidad E, los valores obtenidos son inferiores en los aceros inoxidables respecto al acero al carbono, independientemente de los diámetros de las barras o de su proceso de fabricación. La instrucción EHE-08 establece el valor de $200.000 \mathrm{~N} / \mathrm{mm}^{2}$ para el módulo de elasticidad del acero al carbono, que se cumple en los ensayos realizados, pero que sería aconsejable rebajar a $170.000 \mathrm{~N} / \mathrm{mm}^{2}$ para las armaduras de acero inoxidable a la vista de los resultados, lo que se debe tener en cuenta en los cálculos estructurales del hormigón armado con dichos aceros. Este resultado contrasta con el obtenido por otros autores ${ }^{[19}$ y 20$]$, incluso en el caso de perfiles de acero austenítico laminados en frío ${ }^{[21]}$.

\subsection{Parámetros de ductilidad de las armaduras}

A partir de los resultados de los ensayos de tracción, se han calculado los parámetros de ductilidad de cada armadura cuyos valores medios se resumen en la tabla IV.

Para su comparación, también se recogen en la tabla IV los valores mínimos del ratio $\mathrm{f}_{\mathrm{s}} / \mathrm{f}_{\mathrm{y}} \mathrm{y}$ de $\varepsilon_{\text {máx }}$ que establecen las normas europeas consideradas para los aceros al carbono de alta ductilidad, y los correspondientes valores mínimos del parámetro $\mathrm{p}$ de Cosenza, área $\mathrm{A}_{\text {nom }}$ de Creazza e índice $\mathrm{I}_{\mathrm{d}}$ de Ortega calculados para cada caso.

En términos del ratio de endurecimiento $\mathrm{f}_{\mathrm{s}} / \mathrm{f}_{\mathrm{y}} \mathrm{y}$ de las deformaciones máximas $\varepsilon_{\text {máx }}$, hay diferencias notables entre los tipos de acero y entre los sistemas de fabricación. En el caso de las barras laminadas en caliente, diámetros $\varnothing 16$ y $\varnothing 20$, todos los aceros cumplen con los requisitos mínimos de ductilidad que establecen las normativas consideradas pero, mientras que las barras de acero al carbono se quedan muy

Tabla III. Resultados medios de los ensayos de tracción de los tres aceros, donde $\mathrm{f}_{\mathrm{s}}$ es la carga de rotura, $\mathrm{f}_{\mathrm{y}}$ la tensión en el límite elástico, $\mathrm{E}$ el módulo de elasticidad, $\varepsilon_{\mathrm{y}}$ la deformación unitaria en el límite elástico, $\varepsilon_{\text {máx }}$ la deformación máxima y $\varepsilon_{\mathrm{sh}}$ la deformación al final del escalón de cedencia

Table III. Averages results of tensile tests of the three steels, being $f_{s}$ is the ultimate tensile strength, $f_{y}$ yield strength, $E$ modulus of elasticity, $\varepsilon_{y} 0.2 \%$ proof stress, $\varepsilon_{\max }$ ultimate tensile strain and $\varepsilon_{\text {sh }}$ the plastic plateau deformation

\begin{tabular}{lrrrrrrc}
\hline Acero & $\begin{array}{c}\boldsymbol{\varnothing} \\
\mathbf{m m}\end{array}$ & $\begin{array}{c}\mathbf{f}_{\mathbf{s}} \\
\mathbf{N} / \mathbf{m m}^{\mathbf{2}}\end{array}$ & $\begin{array}{c}\mathbf{f}_{\mathbf{y}} \\
\mathbf{N} / \mathbf{m m}^{\mathbf{2}}\end{array}$ & $\begin{array}{c}\mathbf{E} \\
\mathbf{N} / \mathbf{m m}^{\mathbf{2}}\end{array}$ & $\begin{array}{c}\varepsilon_{\mathbf{y}} \\
\mathbf{\%}\end{array}$ & $\begin{array}{c}\varepsilon_{\mathbf{m a ́ x}} \\
\mathbf{\%}\end{array}$ & $\begin{array}{c}\varepsilon_{\mathbf{s h}} \\
\mathbf{\%}\end{array}$ \\
\hline B500SD & 8 & 875 & 684 & 216.000 & 0,60 & 8,37 & - \\
& 16 & 736 & 602 & 207.000 & 0,54 & 13,32 & 1,50 \\
AISI 304 & 20 & 671 & 556 & 206.000 & 0,51 & 11,84 & 1,09 \\
& 8 & 1.060 & 1.027 & 145.000 & 1,14 & 5,82 & - \\
AISI 2304 & 16 & 743 & 548 & 174.000 & 0,55 & 18,68 & - \\
& 20 & 728 & 507 & 179.000 & 0,52 & 30,12 & - \\
& 16 & 1.066 & 1.003 & 189.000 & 0,85 & 7,02 & - \\
& 20 & 711 & 529 & 179.000 & 0,53 & 24,46 & - \\
& 747 & 609 & 184.000 & 0,57 & 25,62 & - \\
\hline
\end{tabular}


Tabla IV. Parámetros de ductilidad experimentales de las armaduras, valores mínimos que establecen las diferentes normativas europeas y valores calculados según el criterio de acero equivalente para los aceros de ductilidad alta

Table IV. Experimental parameters of the reinforcements ductility, minimun values that establish the various European standards and calculated values considering the concept of equivalent steel for high ductility steels

\begin{tabular}{|c|c|c|c|c|c|c|}
\hline Acero & $\underset{\mathrm{mm}}{\varnothing}$ & $\mathbf{f}_{s} / \mathbf{f}_{y}$ & $\begin{array}{c}\varepsilon_{\text {máx }} \\
\%\end{array}$ & p & $\begin{array}{c}A_{\text {nom }} \\
\mathrm{N} / \mathrm{mm}^{2}\end{array}$ & $I_{d}$ \\
\hline \multirow[t]{3}{*}{ B500SD } & 8 & 1,28 & 8,37 & 1,56 & 1.025 & 58 \\
\hline & 16 & 1,22 & 13,32 & 2,24 & 1.160 & 99 \\
\hline & 20 & 1,21 & 11,84 & 1,85 & 885 & 94 \\
\hline \multirow[t]{3}{*}{ AISI 304} & 8 & 1,03 & 5,82 & 0,17 & 113 & 15 \\
\hline & 16 & 1,36 & 18,68 & 3,55 & 2.383 & 138 \\
\hline & 20 & 1,44 & 30,12 & 6,11 & 4.381 & 257 \\
\hline \multirow[t]{3}{*}{ AISI 2304} & 8 & 1,06 & 7,02 & 0,36 & 271 & 25 \\
\hline & 16 & 1,34 & 24,46 & 4,22 & 2.937 & 193 \\
\hline & 20 & 1,23 & 25,62 & 2,98 & 2.321 & 170 \\
\hline Norma & $\begin{array}{c}\text { Alta } \\
\text { ductilidad }\end{array}$ & $\mathbf{f}_{s} / \mathbf{f}_{y}$ & $\underset{\%}{\varepsilon_{\operatorname{máx}}}$ & p & $\begin{array}{c}\mathrm{A}_{\mathrm{nom}}{ }^{*} \\
\mathrm{~N} / \mathrm{mm}^{2}\end{array}$ & $\mathrm{I}_{\mathrm{d}}^{*}$ \\
\hline EHE 08 & SD & $1,20-1,35$ & 7,5 & $1,06-1,76$ & 362,50 & $63,8-68,15$ \\
\hline CM 90 & $S$ & 1,15 & 6,0 & 0,70 & 287,50 & 49,45 \\
\hline EC 2 & $\mathrm{H}$ & 1,08 & 5,0 & 0,34 & 237,50 & 39,52 \\
\hline \multirow[t]{2}{*}{$\mathrm{EC} 8\left(^{* *}\right)$} & $\mathrm{DCH}$ & $1,20-1,35$ & 9,0 & $1,22-2,02$ & 437,50 & $77,0-82,25$ \\
\hline & & 1,13 & 9,0 & 0,83 & 437,50 & 74,55 \\
\hline \multirow[t]{2}{*}{ CEB } & $S$ & 1,15 & 6,0 & 0,70 & 287,50 & 49,45 \\
\hline & & 1,17 & 5,0 & 0,68 & 237,50 & 41,23 \\
\hline
\end{tabular}

cerca de dichos límites mínimos, siendo el ratio de endurecimiento de 1,21 y 1,22 frente al límite mínimo de 1,20 que establece la EHE-08, las barras de acero inoxidable los superan muy por encima, alcanzando valores de hasta 1,44; especialmente las deformaciones máximas, que en el caso de los diámetros $\varnothing 20$, los aceros inoxidables llegan a duplicar a las del acero al carbono: $25,62 \%$ y $30,12 \%$ frente a $11,84 \%$.

En cambio, para las barras laminadas en frío, diámetros $\varnothing 8$, se comprueba que, mientras las de acero B500SD cumplen con todos los requisitos de ductilidad, las armaduras de acero inoxidable sólo se acercan a los límites del Eurocódigo 2, que son los menos exigentes.

En cuanto a los criterios de ductilidad según el concepto de acero equivalente (Tabla IV), los aceros inoxidables consiguen índices muy superiores a los del acero al carbono, a excepción nuevamente de las barras laminadas en frío, que alcanzan altas resistencias con escasas deformaciones.

En el caso particular del diámetro Ø20 del acero inoxidable AISI 304, el valor medio del parámetro $\mathrm{p}$ obtenido es de 6,11 , lo que supone tener una capacidad de rotación $6,11 / 1,85=3,3$ veces superior a la barra similar del acero al carbono. En cambio, las barras de acero inoxidable AISI 304 laminadas en frío tienen un parámetro p de Cosenza medio de 0,17 frente a 1,56 de sus homólogas de acero al carbono, es decir, proporcionan una capacidad de rotación 9,2 veces inferior.

Si se analiza la clasificación de ductilidad según la propuesta del $\mathrm{CEB}^{[15]}$, los resultados se recogen en la figura 2.

La línea discontinua establece el límite mínimo de valores para los aceros de alta ductilidad tipo "S" (según las Ec. (4-6)). Las dos armaduras que quedan fuera de la línea discontinua corresponden a los diámetros de las barras de acero inoxidable laminadas en frío. Respecto a los aceros inoxidables laminados en caliente, todos los diámetros quedan claramente a la derecha de sus homólogos de acero al carbono, demostrando su mayor ductilidad. 


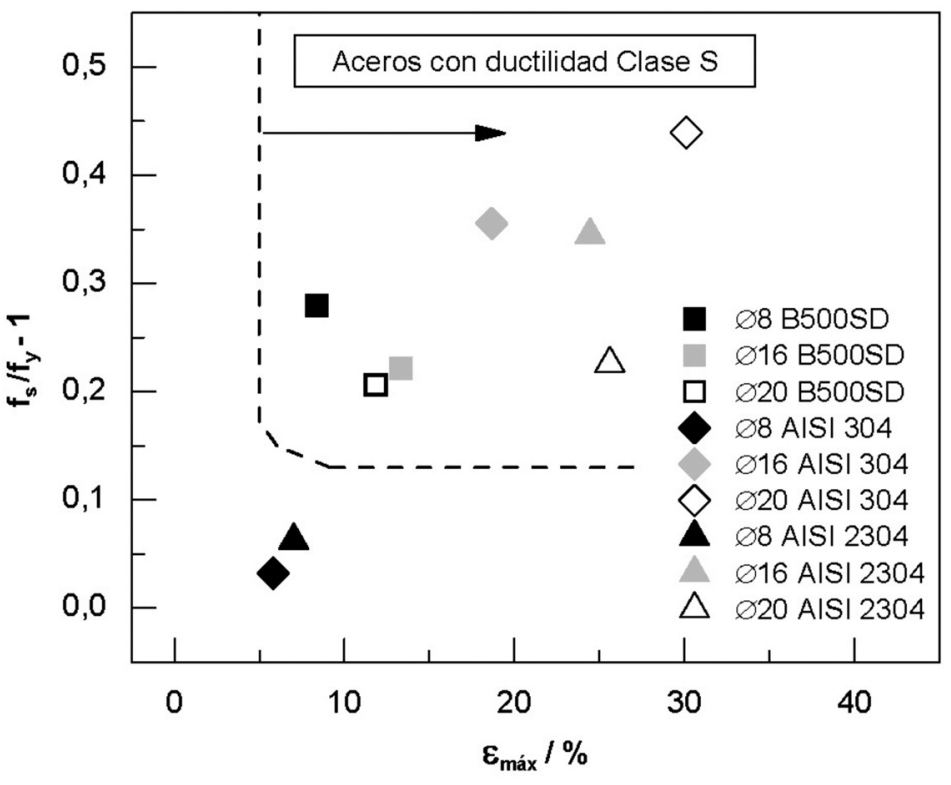

Figura 2. Clasificación de ductilidad del acero tipo S propuesta por el CEB y los parámetros obtenidos del acero al carbono B500SD y los aceros inoxidables AISI 304 y 2304.

Figure 2. Classification of type $S$ steel ductility proposed by the CEB and the parameters obtained for carbon steel B500SD and stainless steels AISI 304 and 2304.

\subsection{Cálculos a nivel de sección}

A partir de los resultados de los ensayos a tracción de cada uno de los aceros, se han calculado los dia- gramas $\mathrm{M}-\varphi$ relativos de las secciones de vigas planas y de canto consideradas en el estudio, armadas con diferentes cuantías de acero al carbono y de acero inoxidable dúplex, que se representan en la figura 3 .

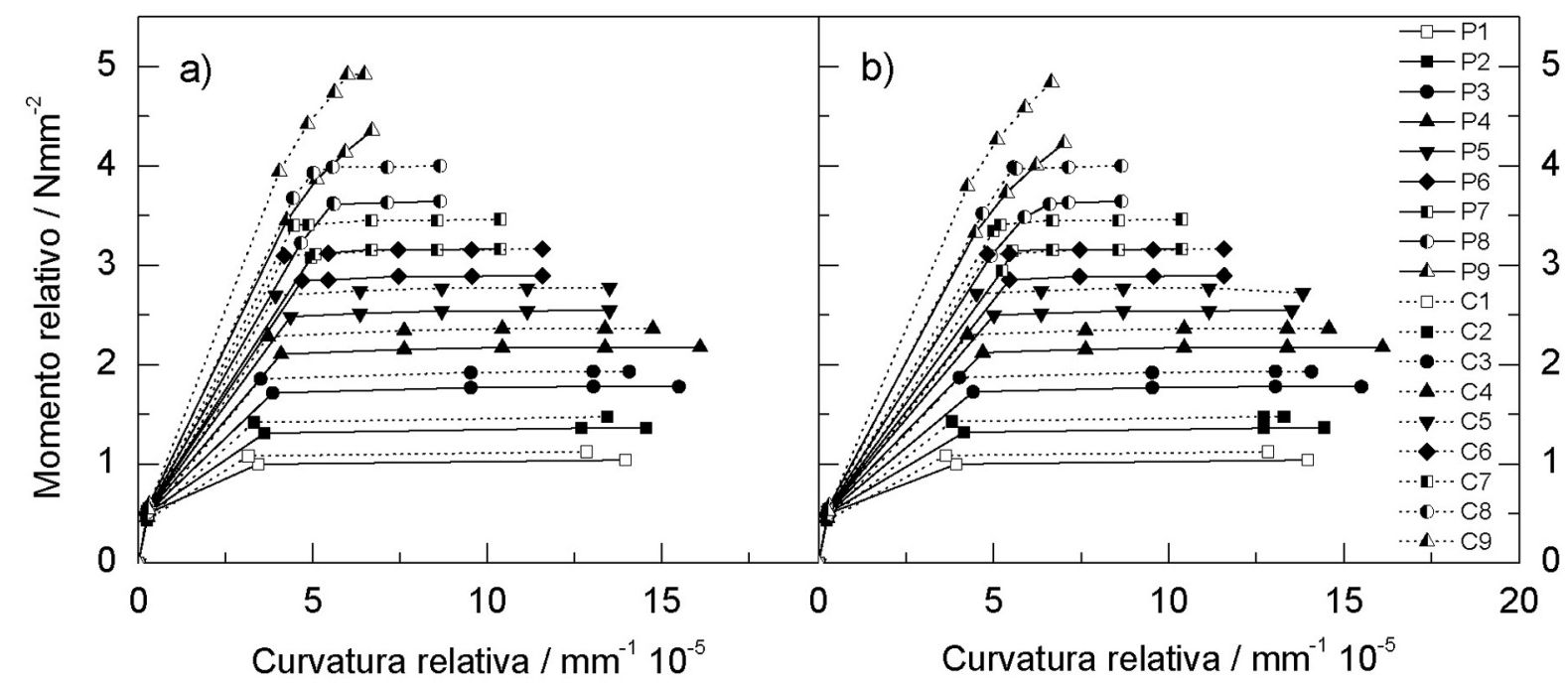

Figura 3. Diagramas $\mathrm{M}-\varphi$ relativos de las secciones de vigas armadas con diferentes cuantías de: a) acero al carbono y b) acero inoxidable dúplex.

Figure 3. M- $\varphi$ relationships diagrams of reinforced concrete sections of beams with different amounts of: a) carbon steel and b) duplex stainless steel. 
Se denomina momento relativo a la relación $\mathrm{M} / \mathrm{b} \cdot \mathrm{h}^{2}$ y curvatura relativa al producto $\varphi \cdot h$, conceptos que permiten representar en un mismo diagrama secciones de diferentes dimensiones de ancho por canto $(b \cdot h)$.

A partir de los diagramas $M-\varphi$, en la tabla V se recogen los resultados de curvatura elástica $\varphi_{\mathrm{e}}$, curvatura última $\varphi_{\mathrm{u}}$ y ductilidad de la sección $\mathrm{D}_{\mathrm{s}}$ (que se define por el cociente $\varphi_{\mathrm{u}} / \varphi_{\mathrm{e}}$ ), para el armado con acero al carbono y con acero inoxidable.

Los valores de ductilidad de sección son casi siempre mayores en las secciones armadas con acero al carbono que en las armadas con acero inoxidable, con incrementos de 0,01 a 0,57 unidades, es decir, hasta un $17 \%$ de mejora en el caso de las vigas P-2 y C-2.

La superior ductilidad de sección con las armaduras de acero al carbono es consecuencia de la mayor curvatura elástica que proporciona a la sección el acero inoxidable, debido a su menor módulo de elasticidad, y de mantener iguales en ambos aceros los valores de las curvaturas últimas, por estar limitadas a la deformación máxima del acero del 10 \% según la instrucción EHE-08.

El acero inoxidable, pese a ser un material más dúctil en comparación con el acero al carbono, proporciona secciones de hormigón armado menos dúctiles. La superior ductilidad del acero inoxidable respecto al acero al carbono se manifiesta para unas deformaciones que quedan fuera del cálculo estructural convencional, es decir, que no se puede aprovechar para aspectos tales como la redistribución de momentos, pero que tiene una ventaja importante en el caso de colapso de la estructura. Para este caso, se podría establecer el concepto de una "curvatura última de rotura $\varphi_{u r}$ " de la sección que tuviera en cuenta una mayor deformación máxima del acero, a partir de la cual

Tabla V. Curvaturas elástica $\varphi_{e}$ y última $\varphi_{u}$, y ductilidad de la sección $D_{s}$ de las vigas armadas con acero al carbono y acero inoxidable dúplex

Table V. Elastic curvature $\varphi_{e}$, last curvature $\varphi_{u}$ and ductility of section $D_{s}$ of reinforced beams with carbon steel and duplex stainless steel

\begin{tabular}{|c|c|c|c|c|c|c|c|}
\hline \multirow[b]{2}{*}{ Viga } & & \multicolumn{3}{|c|}{ Acero al carbono } & \multicolumn{3}{|c|}{ Acero inoxidable dúplex } \\
\hline & & $\underset{\mathrm{mm}^{-1}}{\varphi_{\mathrm{e}}} 1^{-6}$ & $\underset{\mathrm{mm}^{-1}{ }^{-1} 10^{-6}}{\varphi_{u}}$ & $D_{s}=\varphi_{u} / \varphi_{e}$ & $\underset{\mathrm{mm}^{-1} \mathrm{e}_{\mathrm{e}}}{\mathbf{p}^{-6}}$ & $\underset{\mathrm{mm}^{-1}}{\varphi_{\mathrm{u}}} 10^{-6}$ & $D_{s}=\varphi_{u} / \varphi_{e}$ \\
\hline & $\mathrm{P} 1$ & 11,45 & 46,57 & 4,07 & 13,13 & 46,57 & 3,55 \\
\hline & $\mathrm{P} 2$ & 12,05 & 48,53 & 4,03 & 13,86 & 48,10 & 3,47 \\
\hline & P3 & 12,83 & 51,67 & 4,03 & 14,72 & 51,67 & 3,51 \\
\hline Plana & P4 & 13,64 & 53,69 & 3,94 & 15,64 & 53,69 & 3,43 \\
\hline $50 \times 30$ & P5 & 14,53 & 45,04 & 3,10 & 16,69 & 45,04 & 2,70 \\
\hline \multirow[t]{7}{*}{$\mathrm{cm}$} & P6 & 15,63 & 38,61 & 2,47 & 18,16 & 38,61 & 2,13 \\
\hline & $\mathrm{P} 7$ & 16,89 & 34,59 & 2,05 & 18,43 & 34,59 & 1,88 \\
\hline & P8 & 18,70 & 28,83 & 1,54 & 22,00 & 28,83 & 1,31 \\
\hline & P9 & $14,18^{*}$ & 22,33 & 1,58 & $14,92^{*}$ & 23,37 & 1,57 \\
\hline & C1 & 6,30 & 25,69 & 4,08 & 7,28 & 25,69 & 3,53 \\
\hline & $\mathrm{C} 2$ & 6,61 & 26,88 & 4,07 & 7,61 & 26,59 & 3,49 \\
\hline & C3 & 7,01 & 28,15 & 4,02 & 8,05 & 28,15 & 3,50 \\
\hline De canto & C4 & 7,42 & 29,51 & 3,98 & 8,51 & 29,15 & 3,43 \\
\hline $30 \times 50$ & C5 & 7,86 & 27,03 & 3,44 & 9,02 & 27,66 & 3,07 \\
\hline \multirow[t]{4}{*}{$\mathrm{cm}$} & C6 & 8,37 & 23,16 & 2,77 & 9,64 & 23,16 & 2,40 \\
\hline & C7 & 8,88 & 20,76 & 2,34 & 10,39 & 20,76 & 2,00 \\
\hline & C8 & 10,02 & 17,30 & 1,73 & 11,29 & 17,30 & 1,53 \\
\hline & $\mathrm{C9}$ & 12,00 & 12,97 & 1,08 & $8,50^{*}$ & 13,29 & 1,56 \\
\hline
\end{tabular}

* En estos casos, el acero no alcanza su límite de fluencia. Esta curvatura se obtiene cuando el hormigón alcanza una deformación máxima del $2 \%$. 
se obtendría una "ductilidad en rotura de la sección $\mathrm{D}_{\text {sr }}$ " que seguramente destacaría las excelentes cualidades de ductilidad de las armaduras de acero inoxidable.

\subsection{Ensayos a flexión de las vigas}

Los resultados de los ensayos a flexión de las cuatro vigas se resumen en la tabla VI, donde $f_{c, \text { real }}$ es la resistencia real del hormigón obtenida para las probetas elaboradas al fabricar las vigas, $Q_{\text {máx }}$ es la fuerza de rotura máxima desarrollada por la máquina en el ensayo, $M_{\text {máx }}$ es el momento flector de rotura en el centro del vano $\left(\mathrm{M}_{\text {máx }}=0,5 \cdot \mathrm{Q}_{\text {máx }} \cdot \ell / 3\right), \delta_{\text {máx }}$ es la flecha máxima marcada por la prensa, $h_{w}$ es la altura de fisura más alta encontrada, medida desde la cara inferior de la viga y $\mathrm{s}_{\mathrm{m}}$ es la separación media entre fisuras.

En la figura 4 se recoge el diagrama carga-deformación de las cuatro vigas, donde se aprecia cómo el comportamiento de la viga 1 armada con 2 barras corrugadas de diámetro $\varnothing 8$ de acero al carbono es más dúctil que su homóloga armada con acero inoxidable dúplex, aunque alcanza menor carga máxima. Sin embargo, el comportamiento es muy similar entre las vigas armadas con dos barras corrugadas de diámetro $\varnothing 16$, aunque la rotura de la viga

Tabla VI. Resultados de los ensayos de flexión de las cuatro vigas elaboradas Table VI. Results of bending tests of the four beams produced

\begin{tabular}{|c|c|c|c|c|c|c|c|}
\hline Viga & $\begin{array}{c}\text { Armadura } \\
\text { y tipo de acero }\end{array}$ & $\begin{array}{r}\mathbf{f}_{\mathrm{c}, \text { real }} \\
\mathrm{N} / \mathrm{mm}^{2}\end{array}$ & $\underset{\mathbf{k N}}{Q_{\operatorname{máx}}}$ & $\begin{array}{l}\mathbf{M}_{\text {máx }} \\
\mathbf{k N m}\end{array}$ & $\begin{array}{l}\delta_{\text {máx }} \\
\mathrm{mm}\end{array}$ & $\underset{\mathrm{mm}}{\mathrm{h}_{\mathrm{w}}}$ & $\underset{\mathbf{m m}}{\mathbf{s}_{\mathrm{m}}}$ \\
\hline 1 & $2 \varnothing 8$ B500SD & 26,57 & 47,46 & 7,91 & 12,9 & 100 & 100 \\
\hline 2 & $2 \varnothing 16$ B500SD & 27,37 & 98,93 & 16,48 & 6,3 & 54 & 110 \\
\hline 3 & 2 Ø8 AISI 2304 & 26,57 & 68,21 & 11,37 & 12,6 & 95 & 97 \\
\hline 4 & $2 \varnothing 16$ AISI 2304 & 27,37 & 103,72 & 17,28 & 6,7 & 70 & 72 \\
\hline
\end{tabular}

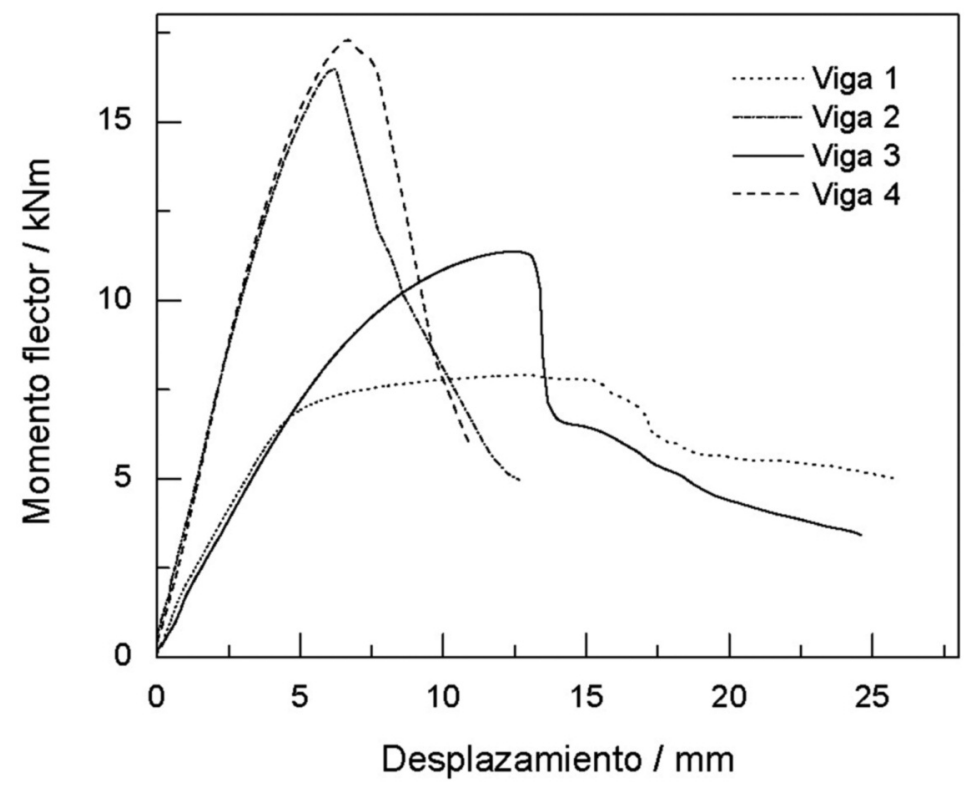

Figura 4. Diagramas carga-deformación de las cuatro vigas ensayadas.

Figure 4. Load-deflection diagrams of the four beams tested. 
Tabla VII. Flecha $(\delta)$, abertura $\left(w_{k}\right)$ y separación media entre fisuras $\left(\mathrm{s}_{\mathrm{m}}\right)$ según cálculo teórico de acuerdo a la EHE-08

Table VII. Deflection $(\delta)$, opening $\left(w_{k}\right)$ and average distance between cracks $\left(s_{m}\right)$ as theoretical calculation according to EHE-08

\begin{tabular}{lcccc}
\hline Viga & $\begin{array}{c}\mathbf{Q} \\
\mathbf{k N}\end{array}$ & $\begin{array}{c}\boldsymbol{\delta} \\
\mathbf{m m}\end{array}$ & $\begin{array}{c}\mathbf{w}_{\mathbf{k}} \\
\mathbf{m m}\end{array}$ & $\begin{array}{c}\mathbf{s}_{\mathbf{m}} \\
\mathbf{m m}\end{array}$ \\
\hline 1 & 40 & 0,3 & 0,29 & 56 \\
2 & 90 & 0,1 & 0,13 & 44 \\
3 & 55 & 0,3 & 0,48 & 56 \\
4 & 95 & 0,1 & 0,17 & 44 \\
\hline
\end{tabular}

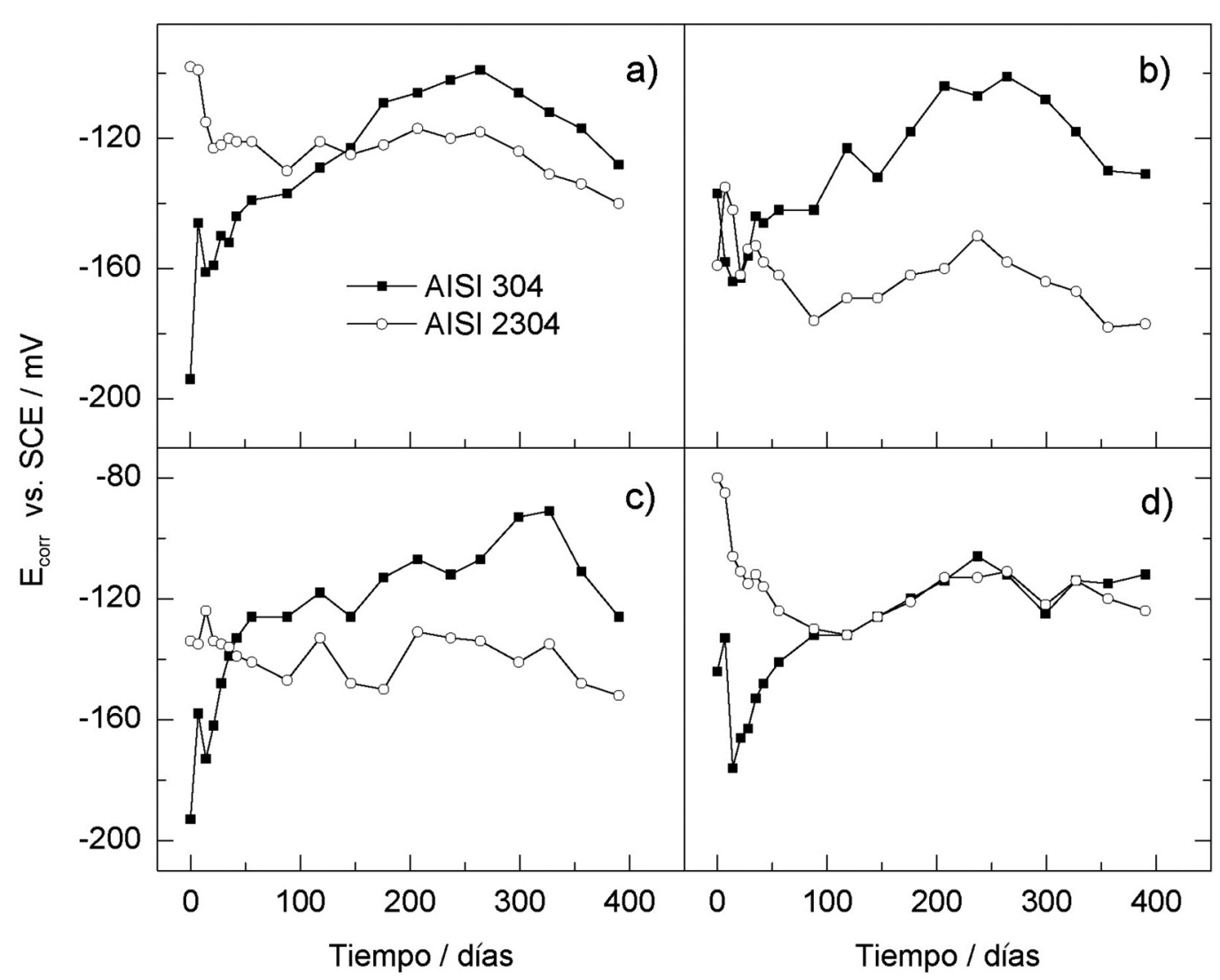

Figura 5. Evolución de los valores de $\mathrm{E}_{\text {corr }}$ de las barras corrugadas de los aceros inoxidables AISI 304 y AISI 2304 embebidas en mortero con a) 0\%; b) 0,4 \%; c) $2 \%$ y d) $4 \%$ de $\mathrm{CaCl}_{2}$.

Figure 5. Evolution of the $E_{\text {corr }}$ values of stainless steels AISI 304 and AISI 2304 reinforcements embedded in OPC mortar with: a) $0 \%$; b) $0.4 \%$; c) $2 \%$ and d) $4 \%$ of $\mathrm{CaCl}_{2}$.

armada con acero inoxidable dúplex es más progresiva.

Los cálculos teóricos de flecha instantánea $\delta$ y abertura de fisura característica $\mathrm{w}_{\mathrm{k}}$, utilizando la formulación recogida en la instrucción EHE-08, realizados para una carga $Q$ registrada durante el ensayo, arrojan los resultados recogidos en la tabla VII. 


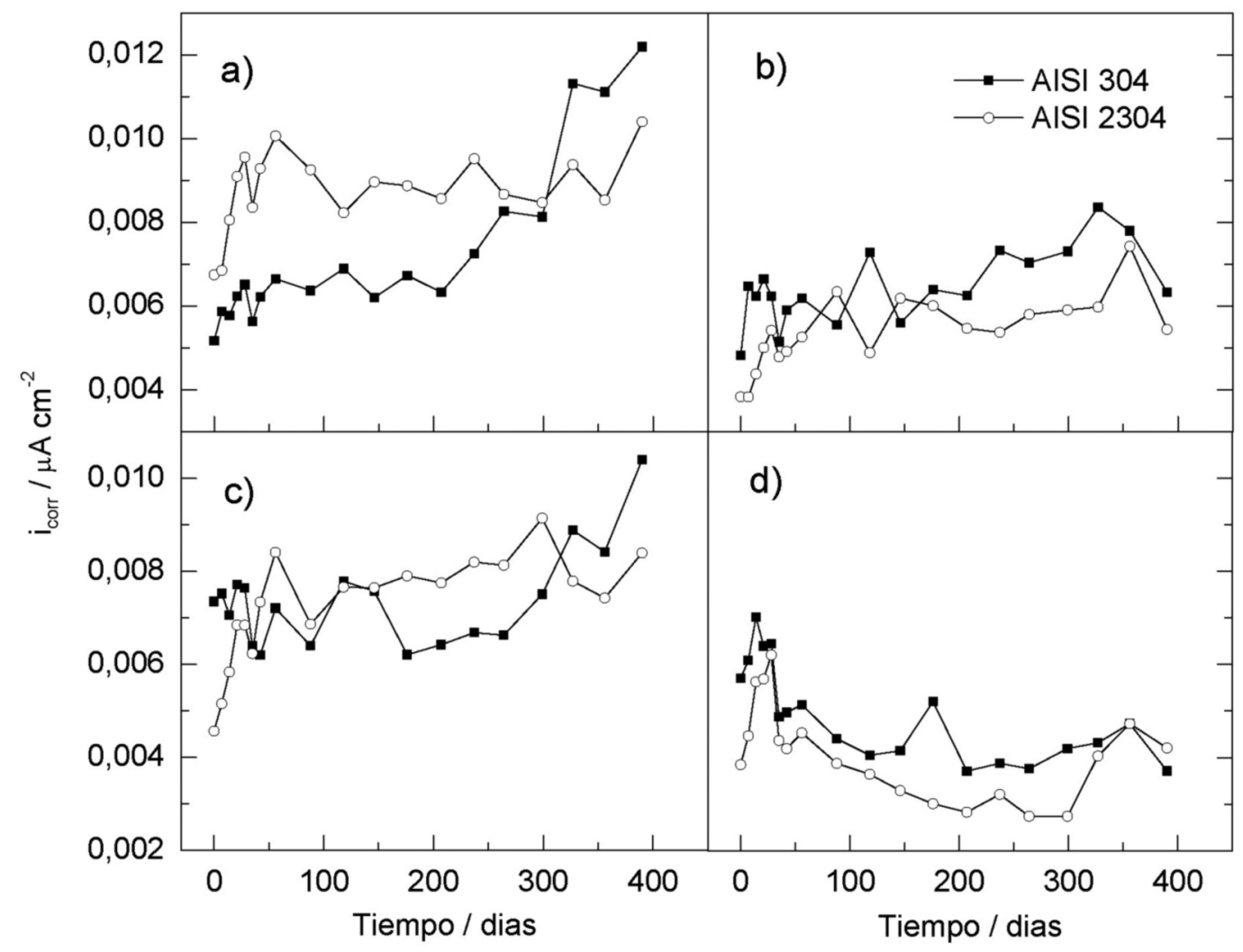

Figura 6. Evolución de los valores de $\mathrm{i}_{\text {corr }}$ obtenidos por el método de resistencia de polarización lineal de las barras corrugadas de los aceros AISI 304 y AISI 2304 embebidas en mortero con a) $0 \%$; b) $0,4 \%$; c) $2 \%$ y d) $4 \%$ de $\mathrm{CaCl}_{2}$.

Figure 6. Evolution of the $i_{\text {corr }}$ values obtained by LPR method for stainless steels AISI 304 and AISI 2304 reinforcements embedded in OPC mortar with: a) $0 \%$; b) $0.4 \%$; c) $2 \%$ and d) $4 \%$ of $\mathrm{CaCl}_{2}$.

Tanto las flechas como las aberturas de fisura teóricas se corresponden con las reales medidas en el ensayo de rotura de las vigas, lo que confirma el comportamiento de los aceros de acuerdo a sus características mecánicas obtenidas en los ensayos de tracción (según se ha observado en la tabla III). En cambio, la separación media entre fisuras teóricas se corresponde aproximadamente con la mitad de las medidas en el ensayo, pero esta circunstancia sólo se puede relacionar con las características del hormigón y el tamaño de las vigas, ya que las propiedades del acero no intervienen en el cálculo teórico.

\subsection{Resistencia a la corrosión de los aceros inoxidables}

La evolución durante el primer año de los valores de $\mathrm{E}_{\text {corr }}$ de los dos aceros inoxidables estudiados, austenítico y dúplex, embebidos en mortero con diferentes cantidades de cloruro cálcico se muestra en la figura 5.

El comportamiento de los dos aceros inoxidables es muy similar entre sí. El potencial de corrosión, después de formarse la capa pasiva en ambos aceros, se estabiliza en una franja de valores comprendidos entre $-120 \mathrm{mV}$ y $-160 \mathrm{mV}$, para la que se considera una probabilidad incierta o inferior al $10 \%$ para que se presente la corrosión del metal cuando se trata de acero al carbono ${ }^{[22]}$.

La evolución de la intensidad de corrosión $\mathrm{i}_{\text {corr' }}$, obtenida mediante el método de resistencia de polarización lineal, de los dos aceros inoxidables estudiados, durante el primer año de vida de las probetas de mortero, para cada una de las cantidades de cloruro cálcico, se recoge en la figura 6 .

Los valores de $i_{\text {corr }}$ son muy similares entre los dos aceros inoxidables y para cualquier contenido de cloruros en el mortero. La intensidad de corro- 


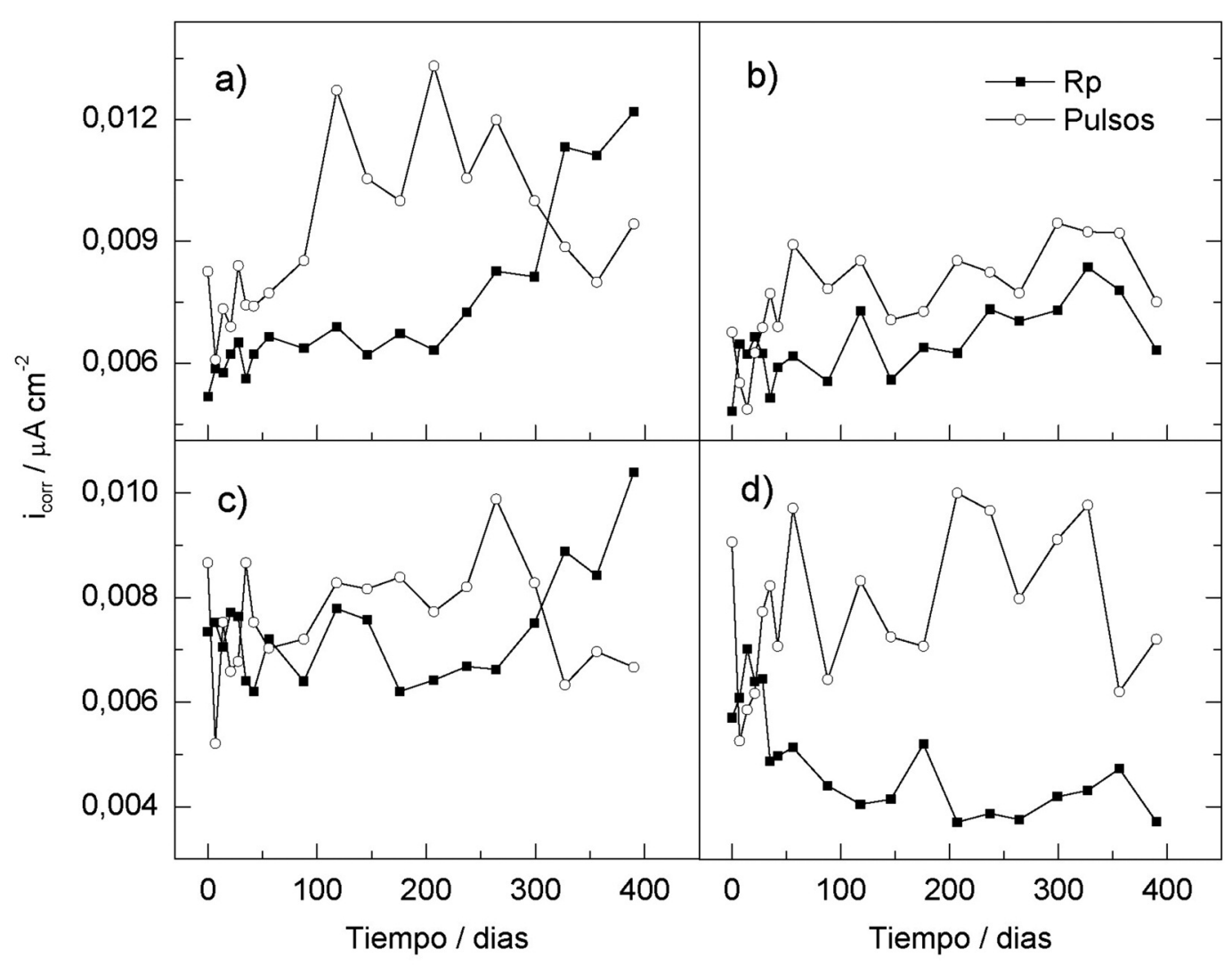

Figura 7. Comparación entre los valores de $\mathrm{i}_{\text {corr }}$ obtenidos por el método de resistencia de polarización lineal y por la técnica de pulsos potenciostáticos, para las barras corrugadas de acero austenítico AISI 304 embebidas en mortero con a) $0 \%$; b) $0,4 \%$; c) $2 \%$ y d) $4 \%$ de $\mathrm{CaCl}_{2}$.

Figure 7. Comparison between $i_{\text {corr }}$ values obtained by LPR method and by potentiostatic pulses technique, for stainless steels AISI 304 reinforcements embedded in OPC mortar with: a) $0 \%$; b) $0.4 \%$; c) $2 \%$ and d) $4 \%$ of $\mathrm{CaCl}_{2}$.

sión se encuentra muy por debajo del límite de $0,1 \mu \mathrm{A} / \mathrm{cm}^{2}$, lo que indica una corrosión insignificante en términos de vida útil de la estructura, es decir, los dos aceros se encuentran en estado pasivo, incluso para el contenido del $4 \%$ de cloruros en el mortero y transcurrido un tiempo de exposición de un año, resultado similar al de otros estudios ${ }^{[23-26]}$.

En la figura 7 se comparan los valores de $\mathrm{i}_{\text {corr }}$ obtenidos por el método de resistencia de polarización lineal y por la técnica de pulsos potenciostáticos, de las barras corrugadas de acero inoxidable austenítico AISI 304, para cada uno de los contenidos de cloruro cálcico considerados en el estudio.

En la figura 8 se hace la misma comparación para el acero dúplex AISI 2304.

Los valores de $i_{\text {corr }}$ obtenidos para los dos aceros inoxidables por ambos métodos son similares entre sí, con diferencias máximas entre técnicas que apenas superan los $6 \cdot 10^{-3} \mu \mathrm{A} / \mathrm{cm}^{2}$, mostrando para tiempos de larga exposición en hormigones muy contaminados con cloruros una pasividad estable de las armaduras, con lo que la durabilidad esperable de la estructura respecto a su armado con acero al carbono será mucho mayor.

\section{CONCLUSIONES}

- Las armaduras de acero inoxidable estudiadas, la austenítica AISI 304 y la dúplex AISI 2304, se diferencian de las del acero al carbono B500SD en dos características mecánicas importantes; por un lado presentan una ductilidad muy superior cuando las barras son laminadas en caliente pero inferior cuando se laminan en frío, y por otro alcanzan un módulo de elasticidad ligeramente inferior, que se puede establecer 


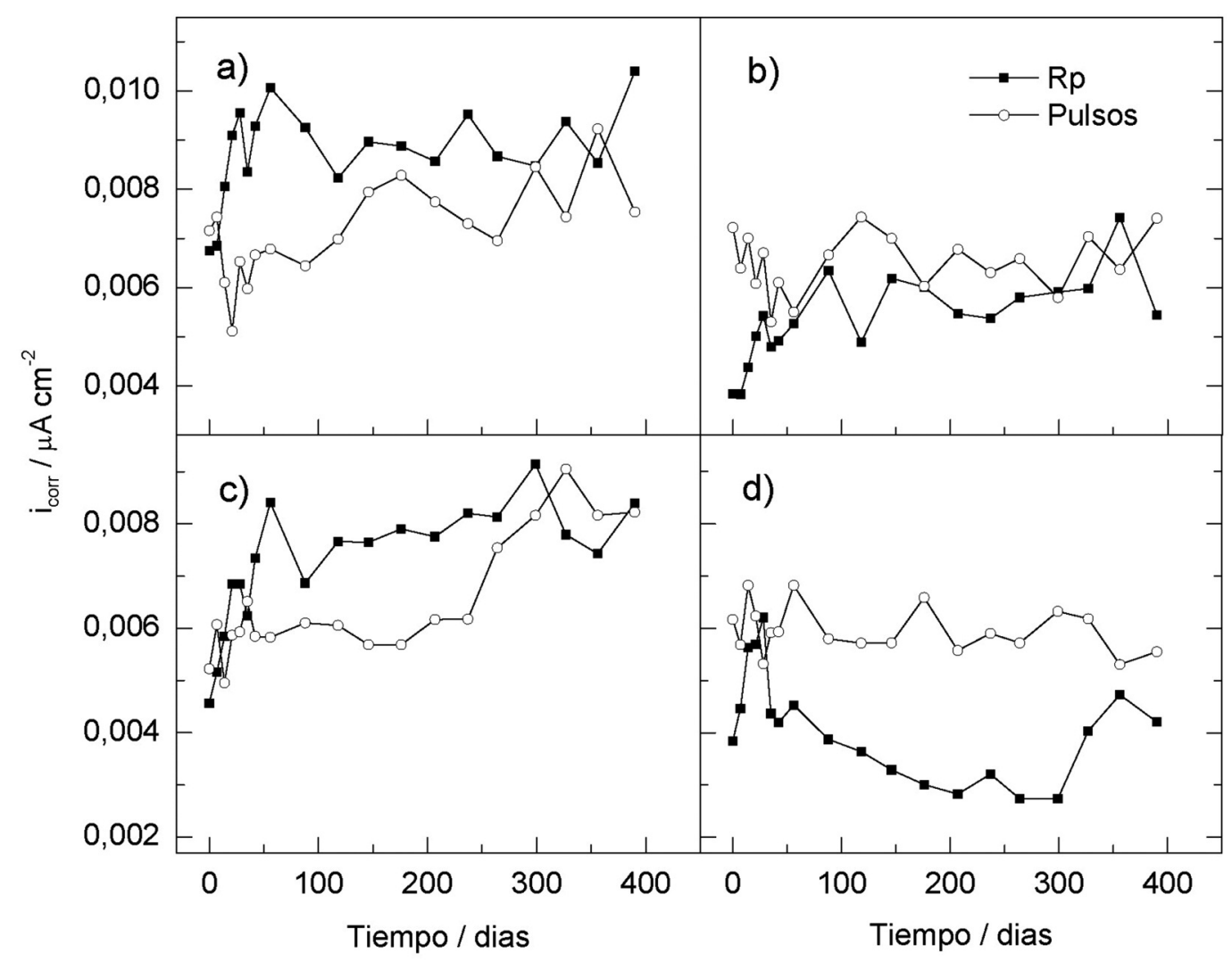

Figura 8. Comparación entre los valores de $i_{\text {corr }}$ obtenidos por el método de resistencia de polarización lineal y por la técnica de pulsos potenciostáticos, para las barras corrugadas de acero dúplex AISI 2304 embebidas en mortero con a) $0 \%$; b) $0,4 \%$; c) $2 \%$ y d) $4 \%$ de $\mathrm{CaCl}_{2}$.

Figure 8. Comparison between $i_{\text {corr }}$ values obtained by LPR method and by potentiostatic pulses technique, of stainless steels AISI 2304 reinforcements embedded in OPC mortar with: a) $0 \%$; b) $0.4 \%$; c) $2 \%$ and d) $4 \%$ of $\mathrm{CaCl}_{2}$.

en $170.000 \mathrm{~N} / \mathrm{mm}^{2}$, condicionando su comportamiento a nivel de sección y a nivel de pieza o elemento estructural, por lo que se recomienda tener en cuenta este valor del módulo de elasticidad en los cálculos estructurales.

- Respecto a la resistencia a la corrosión por cloruros, ninguna de las dos armaduras inoxidables presenta valores de intensidad de corrosión que indiquen inicio de corrosión por picaduras tras embeberlas en morteros con hasta un $4 \%$ de cloruro cálcico y mantenerlas con un $95 \%$ de humedad durante el primer año de vida de las probetas. Las armaduras de acero inoxidable dúplex AISI 2304 son un 20 \% más económicas que las de acero inoxidable austenítico AISI 304 por su menor contenido en níquel, y sin embargo, presentan una resistencia a la corrosión a los cloruros similar.

- La técnica de pulsos potenciostáticos permite determinar la intensidad de corrosión de las barras corrugadas de acero inoxidable embebidas en hormigón, obteniendo valores muy similares a los registrados por la técnica de resistencia de polarización lineal, con la ventaja de que no se necesita conocer cúal es el área expuesta de la barra y de ser una técnica no destructiva, por lo que se puede aplicar a las estructuras in situ.

\section{Agradecimientos}

Los autores quieren expresar su gratitud al Plan Nacional de $\mathrm{I}+\mathrm{D}+\mathrm{i}$, por la financiación al Proyecto BIA2011-27182, a Acerinox S.A. y a Roldán S.A. por el suministro de las armaduras de acero inoxidable utilizadas en los ensayos. D. M. Bastidas agradece la financiación al Programa Ramón y Cajal del Ministerio de Economía y Competitividad. 


\section{REFERENCIAS}

[1] A. Knudsen y A. Skoysgaard, Concrete Engineering International 3 (2001) 59-62.

[2] S.M. Alvarez, A. Bautista y F. Velasco, Corros. Sci. 53 (2011) 1.748-1.755.

[3] Highways Agency. Design Manual for Roads and Bridge. Vol. 1. Sec. 3. Part 15: BA 84/02 Use of Stainless Steel Reinforcement in Highway Structures, Londres, Inglaterra, 2002.

[4] G. Gedge, Symposium Structural applications of stainless steel in building and architecture, EUROINOX, Septiembre 2000, Bruselas. (www.euro-inox.org).

[5] S. Qian, D. Qu, y G. Coates, Canadian Metallurgical Quarterly 4 (2006) 475-484.

[6] C.M. Abreu, M.J. Cristóbal, M.F. Montemor, X.R. Nóvoa, G. Pena, y M.C. Pérez, Electrochim. Acta 47 (2002) 2.271-2.279.

[7] M.C. García-Alonso, J.A.González, J. Miranda, M.L. Escudero, M.J. Correia, M. Salta y A. Bennani, Cement. Concrete Res. 37 (2007) 1.562-1.569.

[8] AENOR, UNE-EN 10002-1 Materiales metálicos. Ensayos de tracción. Parte I: Método de ensayo a temperatura ambiente. Madrid, 2002.

[9] AENOR, UNE-EN ISO 15630-1 Aceros para el armado y el pretensado del hormigón. Métodos de ensayo. Parte I: Barras, alambres y alambrón para hormigón armado. Madrid, 2003.

[10] Comisión Permanente Del Hormigón, EHE-08 Instrucción de hormigón estructural. Ministerio de Fomento, Madrid, 2008.

[11] AENOR, UNE-ENV 1992-1-1:EUROCÓDIGO2 Proyecto de estructuras de hormigón. Parte 11: Reglas generales y reglas para edificación. Madrid, 1993.

[12] AENOR, EN 1998-1: EUROCÓDIGO 8 Proyecto de estructuras frente a sismo. Parte 1: Reglas generales, acciones sísmicas y reglas para edificación. Asociación Española de Normalización y Certificación, Madrid, 1998.
[13] GEHO-CEB, Código modelo CEB-FIP 1990. Colegio de Ingenieros de Caminos, Canales y Puertos, Madrid, 1995.

[14] A. Cobo, D.M. Bastidas, M.N. González, E. Medina y J.M. Bastidas, Materiales de Construcción 61 (2011) 613-620.

[15] CEB, Ductility of reinforced concrete structures, Comité Euro-International du Bêton, Bulletin no 242, Stuttgart, 1998.

[16] ASTM G61-86, Standard Test Method for Conducting Cyclic Potentiodynamic Polarization Measurements for Localized Corrosion Susceptibility of Iron-, Nickel-, or Cobalt-Based Alloys, P.A., 2009.

[17] M. Stern y A.L. Geary, J. Electrochem. Soc. 104 (1957) 56-63.

[18] D.M. Bastidas, J.A. González, S. Feliu, A. Cobo y J.M. Miranda, Corrosion 63 (2007) 1.0941.100 .

[19] H. Castro, C. Rodríguez, F.J. Belzunce y A.F. Canteli, J. Mater. Process. Tech. 143-144 (2003) 134-137.

[20] E. Real, C. Rodríguez, F.J. Belzunce y A.F. Canteli, Anales de Mecánica de la Fractura 25 (2008) 367-372.

[21] L. Gardner, A. Talja y N.R. Baddoo, Thin Wall. Struct. 44 (2006) 517-528.

[22] ASTM C876-99. Standard test method for half-cell potentials of uncoated reinforcing steel in concrete. ASTM, 1999.

[23] M.C.García-Alonso, M.L. Escudero, J.M. Miranda, M.I. Vega, F. Capilla, M.J. Correia, M. Salta, A. Bennani y J.A.González, Cement. Concrete Res. 37 (2007) 1.463-1.471.

[24] G. Blanco, A. Bautista y H. Takenouti, Cement. Concrete Comp. 28 (2006) 212-219.

[25] M.Criado, D.M. Bastidas, S. Fajardo, A. Fernández-Jiménez y J.M. Bastidas, Cement. Concrete Comp. 33 (2011) 644-652.

[26] S. Fajardo, D.M. Bastidas, M. Criado, M. Romero y J.M. Bastidas, Constr. Build. Mater. 25 (2011) 4.190-4.196. 